\title{
Complete Photo-fragmentation of the Deuterium Molecule
}

Th. Weber ${ }^{1,3,5}$, A. Czasch ${ }^{1}$, O. Jagutzki ${ }^{1}$, A. Müller ${ }^{1}$, V. Mergel ${ }^{1}$, A. Kheifets ${ }^{2}$,

E. Rotenberg ${ }^{3}$, G. Meigs ${ }^{3}$, M.H. Prior ${ }^{3}$, S. Daveau ${ }^{3}$, A.L. Landers ${ }^{4}$, C.L. Cocke ${ }^{5}$,

T. Osipov ${ }^{5}$, R. Díez Muiño ${ }^{6}$, H. Schmidt-Böcking ${ }^{1}$ and R. Dörner ${ }^{1, *}$

${ }^{1}$ Institut für Kernphysik, Universität Frankfurt, D 60486 Frankfurt, Germany

${ }^{2}$ Research School of Physical Sciences and Engineering, Australian National

University, Canberra ACT 0200, Australia

${ }^{3}$ Lawrence Berkeley National Laboratory, Berkeley CA 94720

${ }^{4}$ Dept. of Physics, Western Michigan University, Kalamazoo, MI 49008

${ }^{5}$ Dept. of Physics, Kansas State University, Manhattan, KS 66506

${ }^{6}$ DIPC and UFM Centro Mixto CSIC-UPV/EHU, 20018 Donostia-San Sebastian, Spain

*Electronic address: doerner@hsb.uni-frankfurt.de

(May 13, 2004)

PACS: (33.60.Cv), (33.80.Eh), (31.15.Ar) 


\begin{abstract}
\end{abstract}
All properties of molecules, from binding and excitation energies to their geometry, are determined by the highly correlated initial state wavefunction of the electrons and nuclei. Perhaps surprisingly, details of these correlations can be revealed by studying the break-up of these systems into their constituents. The fragmentation might be initiated by the absorption of a single photon $[1,2,3,4,5$, 6], collision with a charged particle $[7,8]$ or exposure to a strong laser pulse $[9,10]$. If the exciting interaction is sufficiently understood, one can use the fragmentation process as a tool to learn about the bound initial state $[11,12]$. However, often the interaction and the fragment motions pose formidable challenges to quantum theory $[13,14,15]$.

Here we report the coincident measurement of the momenta of both nuclei and both electrons from the single photon induced fragmentation of the deuterium molecule. The results reveal that the correlated motion of the electrons is strongly dependent on the inter-nuclear separation in the molecular ground state at the instant of photon absorption.

Text

Small systems of Coulomb interacting particles such as the Helium atom or the Hydrogen molecule have been paradigms for quantum theory since its early days. However, despite 80 years of theoretical attention, near exact calculations for such systems are only available for bound states. On the experimental side, the tests of these calculations are largely based upon level energies or single particle momentum 
distributions. Very promising and challenging new classes of experiments are those which achieve a complete description of the outcome following the excitation of the ground state to an unbound continuum. The momenta, i.e. the set of vectors, of all the fragments of an atom or molecule break-up can be measured in coincidence with high precision using state-of-the-art imaging and timing techniques [16]. These asymptotic many-particle momentum distributions are determined by the interaction inducing the fragmentation, the bound initial state from which it emerged, and the interactions between the outgoing particles. Thus it is useful to the experimentalist to keep the interaction process as simple as possible and to choose a geometry where final state interactions are negligible or under control. In the present study we used the absorption of a single photon to fragment the deuterium molecule:

$\mathrm{h} v+\mathrm{D}_{2} \rightarrow 2 \mathrm{e}^{-}+2 \mathrm{~d}^{+}$

Due to their heavy masses, the initial motion of the nuclei in the continuum can be assumed the same as in the ground state at the instant of the electronic transition (Born Oppenheimer approximation). Once the electrons have left the system, the motion of the nuclei is solely determined by their Coulomb repulsion; they accelerate to a Kinetic Energy Release (KER) which corresponds to the Coulomb potential associated with their initial separation. Quantum mechanically one maps the nuclear vibrational wave-function onto the Coulomb potential to yield a KER spectrum. Inverting this process determines the squared nuclear vibrational wave-function from the measured KER spectrum [17]. Furthermore, by selecting events that occur within a fixed subregion in the KER spectrum, one samples molecules for which the corresponding internuclear distance is defined much more precisely than the full extent of the initial nuclear wave-function. This allows us to show how the electronic continuum momentum distribution depends on the inter-nuclear separation in the molecule and its orientation with respect to the photon polarization. 
Multi-particle coincidence experiments have become possible with the advent of modern micro-channel plate multiplier based imaging techniques combined with high resolution time-of-flight measurements [18, 19, 20, 21]. In brief, inside our momentum spectrometer, a supersonic $\mathrm{D}_{2}$-gas jet was crossed with the linear polarized photon beam from the LBNL Advanced Light Source $\left(\mathrm{D}_{2}\right.$ provides a higher target density than a comparable $\mathrm{H}_{2}$ gas jet and data less contaminated by random coincidences from background $\mathrm{H}_{2} \mathrm{O}$ ). The electrons and ions created in the intersection of the photons with the jet are guided by a combination of homogeneous electric and parallel magnetic fields onto channel plate detectors (see www.Roentdek.com). For each particle, the position of impact and the time-of-flight to the detector is registered. From these values and the geometry and fields, the momentum vectors of all particles from each fragmentation event can be calculated. The validity and sensitivity of the mapping of the ground state distribution of inter-nuclear distances to the KER is demonstrated in figure 1. The red line shows the nuclear wave-function obtained when approximating a $\mathrm{D}_{2}$ potential curve by a harmonic oscillator with the correct vibration frequency and equilibrium distance, while the black curve shows the calculation with an improved wave-function obtained from a much more accurate Morse potential.

In figure 2 we illustrate the dependence of the coincident electron angular distribution on the molecular orientation. In this figure the molecular axis, the light polarization axis $\varepsilon$ and one of the electrons are restricted to one plane. The momentum vector of the second electron is fixed perpendicular to that plane pointing towards the observer. By keeping the angle between the two electrons constant at $90^{\circ}$, we expect minimal influence and variation of the electron-electron interaction in the final state. For other geometries we find that the angular distributions are almost completely dominated by the interplay between the electron-electron repulsion and the angular momentum and parity selection rules [12, 22]. 
An atomic photo-ionization cross section can be described by a dipole distribution with its symmetry axis in the direction of the polarization of the incoming photon. In contrast, figure 2 for the molecular case shows a strong dependence of the electron angular distribution on the molecular orientation demonstrating the importance of this new internal reference axis. While the light field attempts to drive electrons toward a dipole pattern keyed to its polarization axis, the two-body Coulomb potential of the molecule tends to favour electrons escaping perpendicular to its axis. Furthermore the distribution is not describable by a pure dipole shape; it shows additional small lobes (see fig. 2b,c) indicating that higher angular momentum components are present. A possible reason for the preferred emission perpendicular to the molecular axis could be the ground state electron momentum distributions in the molecule. Since in configuration space the electronic wave function is elongated along the inter-nuclear axis the momentum space wave function, i.e. the Fourier transform, peaks perpendicular to the molecular axis. The photo-ionization probes the momentum space wave function; indeed, the photo-ionization matrix element in the high energy limits corresponds to the Fourier transform of the initial state [23]. On the other hand, a description of the ionization process in terms of diffraction of an initial outgoing electron by the two centres of the potential can yield similar complex angular distribution patterns. From this one might expect that these emission patterns of the Photo Double Ionization (PDI) shown in figure 2, can be described by the single ionization of a $\mathrm{D}_{2}{ }^{+}$-Ion. Despite these seemingly reasonable qualitative ideas, we show in the following that nature requires something else.

A key result of our experiment is shown in figure 3. The geometry is the same as in figure 2, but here the plots are made for selected regions in the KER spectrum. Surprisingly for the smallest KER (i.e. largest separation) the angular distribution resembles a helium-like dipole pattern, i.e. it is mainly aligned along the polarization axis (see fig. 3a). For the smallest inter-nuclear separation (fig. 3c) the emission pattern 
is essentially orthogonal to the molecular axis and the distribution has changed from a dipole to a four-lobe pattern, indicating higher angular momentum components involved. What is the physical origin of these observations ? First, we exclude interference modulations from simple two centre diffraction, since here the wavelengths of the electrons are 4-6 times the inter-nuclear separation. Second, whereas a multiple scattering of the photoelectron wave at the two centres could lead to a variation of the angular distributions, as is predicted and observed for K-shell ionization of the $\mathrm{CO}$ molecule for instance [24], investigations on $\mathrm{H}_{2}$, applying the method described in [25], show that this effect, as a function of the inter-nuclear separation $\Delta \mathrm{R}$, is small. This is because the protons are relatively weak scattering centres and the long wavelength of the photoelectrons would require long paths within the molecular potential. A remaining possibility might be found in the initial state electronic wave function.

A calculation based on an appropriate two-electron initial state is included in figure $3 \mathrm{~b}$. The experimental data show a mixture of patterns similar to the dipole distribution of figure $3 \mathrm{a}$ and the four lobe structure of figure $3 \mathrm{c}$. A simple model [26, 27] in which a pair of photo ionization amplitudes $f_{\Sigma}$ and $f_{\Pi}$ is introduced for the light polarization parallel $(\Sigma-)$ and perpendicular ( $\Pi-$ transition) to the molecular axis ionizing the molecule is shown as the blue solid line. To evaluate the amplitudes $f_{\Sigma}$ and $f_{\Pi}$ we used a single-centre expansion of the $\mathrm{H}_{2}$ ground state [28], and a Convergent Close-Coupling (CCC) expansion of the final two-electron state in the field of a pointlike charge $Z=2$ [29]. This theory, neglecting two-centre electron-nuclei interaction in the final state, yields only the dipole pattern. The difference between this result and the observations reveals the complex diffraction of the outgoing electron wave from a highly correlated two electron initial state. Further CCC-calculations corresponding to the kinematics of figure $3 \mathrm{a}$ and $3 \mathrm{c}$ based on single-centre Slater type orbitals for different inter-nuclear distances [30] do not differ from the result shown for the equilibrium state in figure $3 \mathrm{~b}$ (not plotted here). Apparently this kind of initial state 
function is not as sensitive to small changes in $\Delta \mathrm{R}$ as the experimental data seem to require.

In conclusion we see complex structures in the electronic angular distribution which depend strongly on the molecular orientation and the inter-nuclear separation. The angular distributions, apparently highly influenced by an appropriate initial state wave function, diffraction and electron-electron correlation, show unexpected behaviour that is not yet understood. Our results are highly sensitive and direct tests of the initial state wavefunction and its correlation effects. An intricate calculation to address our observations is highly desirable. A complete treatment of the break-up of this fundamental molecule would mark a significant step toward understanding quantum dynamics of many particles systems, a subject central to physical and chemical processes.

Acknowledgements This work was supported by DFG, BMBF, and the Chemical Sciences, Geosciences and Biosciences Division, Office of Basic Energy Sciences, Office of Science, U.S. Department of Energy (DOE). The ALS is supported by DOE Contract No. DE-AC03-76SF00098. Th. W. thanks Graduiertenförderung des Landes Hessen, the Alexander von Humboldt Stiftung and the Herrmann Willkomm Stiftung for financial support. We thank Roentdek GmbH (www.Roentdek.com) for support with detectors, and acknowledge very helpful discussion with colleagues M. Walter, J. Briggs, J. Feagin, T. Reddish and V. Schmidt

\section{References}

1. H. Kossmann, O. Schwarzkopf, B. Kämmerling and V. Schmidt, Phys. Rev. Lett., 63, 2040, (1989)

2. G. Dujardin, Phys. Rev. Lett., 35, 5012, (1987) 
3. T.J. Reddish, J.P. Wightman, M.A. MacDonald and S. Cvejanovic, Phys. Rev. Lett., 79, 2438, (1997)

4. J. Wightman, S. Cvejanovic and T.J. Reddish, J. Phys. B, 31, 1753, (1998)

5. D.P. Seccombe, S.A. Collins, T.J. Reddish, P. Selles, L. Malegat, A.K. Kazansky and A. Huetz, J. Phys. B, 35, 3767, (2002)

6. R. Dörner, H. Bräuning, O. Jagutzki, V. Mergel, M. Achler et.al., Phys. Rev. Lett., 81, 5776, (1998)

7. F. Afaneh, R. Dörner, L. Schmidt, Th. Weber, K.E. Stiebing, O. Jagutzki, K.A. Müller and H. Schmidt-Böcking, J. Phys. B., 35, L229, (2002)

8. R.M. Wood, A.K. Edwards, M.F. Steuer, Phys. Rev. A, 4, 1433, (1977)

9. A. Staudte, C.L. Cocke, M.H. Prior, A. Belkacem, C. Ray, H.W. Chong, T.E. Glover, R.W. Schoenlein and U. Saalmann, Phys. Rev. A, 65, 020703, (2002)

10. H. Rottke, C. Trump, M. Wittmann, G. Korn, W. Sandner, R. Moshammer, A. Dorn, C.D. Schröter, D. Fischer, J.R. Crespo Lopez-Urrutia, P. Neumayer, J. Deipenwisch, C. Höhr, B. Feuerstein and J. Ullrich, Phys. Rev. Lett., 89, 013001, (2002)

11. R. Moshammer, J. Ullrich, M. Unverzagt, W. Schmitt, P. Jardin et al. Nucl. Instr. Meth. Phys. Res. Sect. B, 107, 62, (1996)

12. V.G. Levin, V.G. Neudatchin, A.V. Pavlitchankov and Yu.F. Smirnov, J. Phys. $B, \mathbf{1 7}, 1525,(1984)$

13. T.N. Rescigno, M. Baertschy, W.A. Isaacs, C.W. McCurdy, Science, 286, 2474 (1999)

14. Th. Weber, A. Czasch, O. Jagutzki, A. Müller, V. Mergel, A. Kheifets, J. Feagin, E. Rotenberg, G. Meigs, M.H. Prior, S. Daveau, A.L. Landers, C.L. 
Cocke, T. Osipov, H. Schmidt-Böcking and R. Dörner, Phys. Rev. Lett., 92, 163001, (2004)

15. J. Briggs and V. Schmidt, J. Phys. B, 33, R1, (2000)

16. R. Dörner, V. Mergel, L. Spielberger, O. Jagutzki, J. Ullrich, H. SchmidtBöcking, Phys. Rev. A, 57, 1074, (1998)

17. H. Le Rouzo, Phys. Rev. A, 37, 1512, (1988)

18. R. Dörner, V. Mergel, O. Jagutzki, L. Spielberger, J. Ullrich, R. Moshammer, H. Schmidt-Böcking, Physics Reports, 330, 95, (2000)

19. J. Ullrich, R. Moshammer, A. Dorn, R.Dörner, L.Ph.H. Schmidt and H. Schmidt-Böcking, Rep. Prog. Phys., 66, 1463, (2003)

20. Th. Weber, H. Giessen, M. Weckenbrock, A. Staudte, L. Spielberger, O. Jagutzki, V. Mergel, G. Urbasch, M. Vollmer and R. Dörner, Nature, Vol. 405, 8 June, 658, (2000)

21. M. Schulz, R. Moshammer, D. Fischer, H. Kollmus, D.H. Madison, S. Jones, J. Ullrich, Nature, Vol. 422, 6 March, 48, (2003)

22. M. Walter and J.S. Briggs, Phys. Rev. Lett., 85, 1630, (2000)

23. M. Walter and J.S. Briggs, J. Phys. B, 32, 2487, (1999)

24. Th. Weber, M. Weckenbrock, M. Balser, L. Schmidt, O. Jagutzki, W. Arnold, O. Hohn, E. Arenholz, T. Young, T. Osipov, L. Foucar, A. De Fanis, R. Diez Muino, H. Schmidt-Böcking, C.L. Cocke, M.H. Prior and R. Dörner, Phys. Rev. Lett, 90, 153003-1 , (2002)

25. R. Díez Muiño, D. Rolles, F.J.G. de Abajo, C.S. Fadley and M.A.V. Hove, Phys. J. Phys. B, 35, L359, (2002)

26. J.M. Feagin, J. Phys. B, 31, L729, (1998) 
27. T.J. Reddish and J.M. Feagin, J. Phys. B, 32, 2473, (1999)

28. H.W. Joy and R.G. Parr, J. Chem. Phys., 28, 448, (1958)

29. A.S. Kheifets and I. Bray, J. Phys. B, 31, L447, (1998)

30. E.F. Hayes, J. Chem. Phys., 46, 4004, (1967)

Figures:

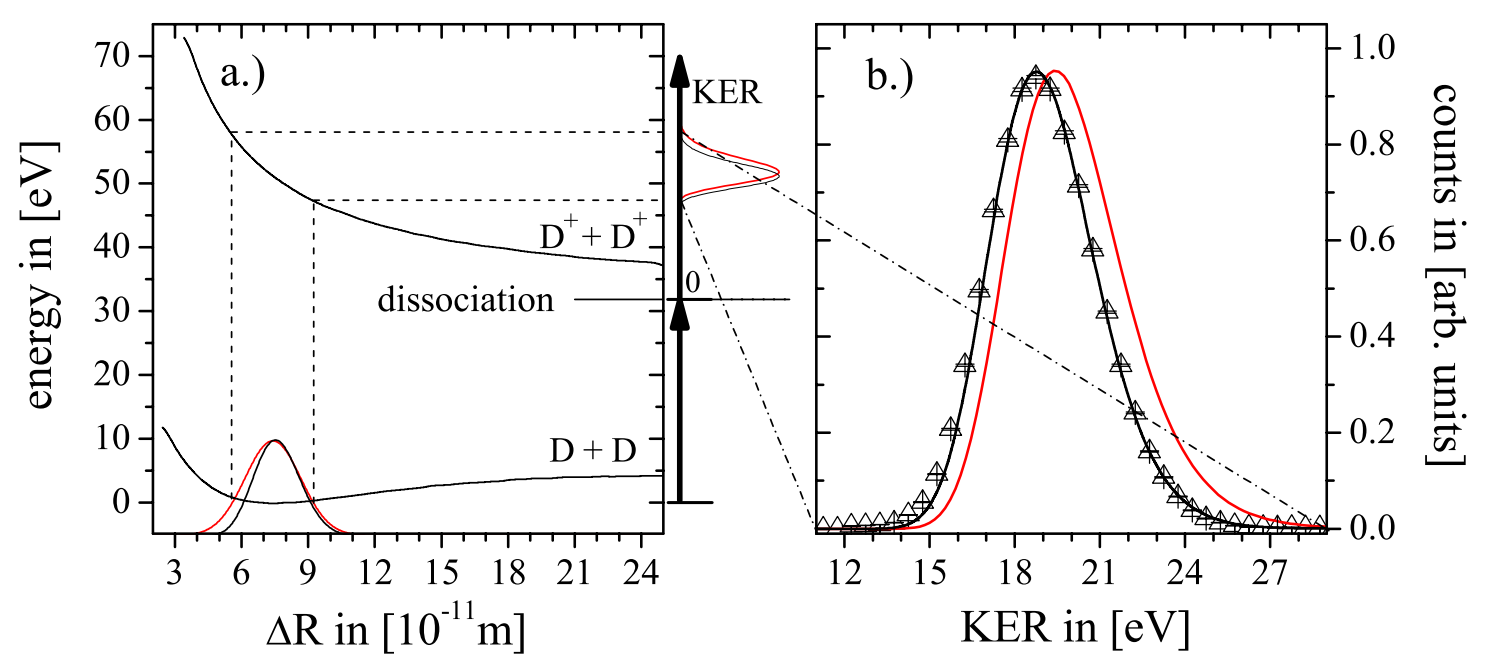

Figure 1: Determination of the inter-nuclear distance $\Delta \mathrm{R}$ in the ground state of $D_{2}$ by measuring the Kinetic Energy Release KER after double ionization with linear polarized light of $75.5 \mathrm{eV}$. The ground state wave function is mirrored at the repulsive $d^{+}+d^{+}$potential curve onto the KER: The red curves show the prediction starting from a harmonic oscillator potential; the black curves show the same from a Morse potential. The open triangles are the experimental measurements. 

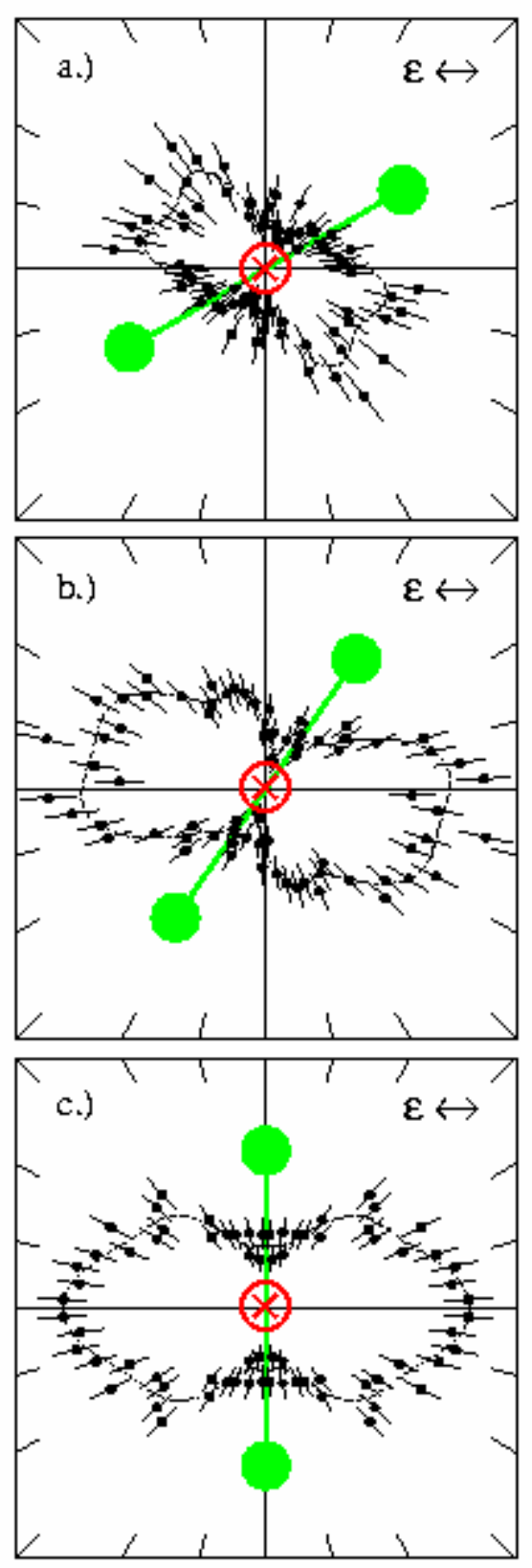

Figure 2: Angular distribution of one electron (black dots) in the plane of the molecular axis (green barbell) and the electric field vector of the linear polarized light $\varepsilon$ (horizontal double arrow) for a photon energy of $75.5 \mathrm{eV}$. The second electron moves orthogonally out of the plane towards the observer (the red 
circled cross). Each electron has $12.25 \mathrm{eV}$ energy. The dashed lines show a fit with spherical harmonics $(l \in[1,4], m \in[0,1])$.

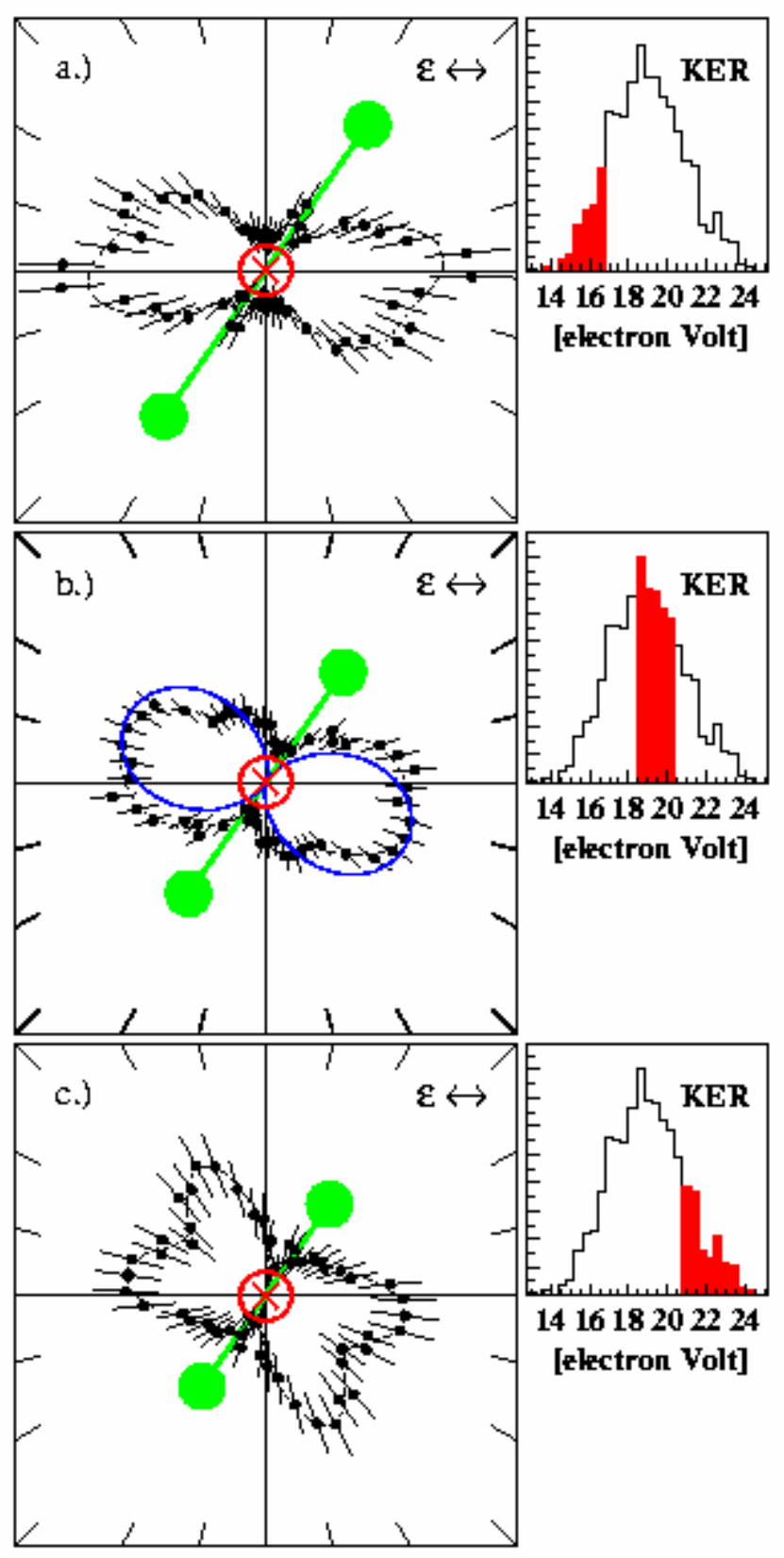


Figure 3: Same as figure 2, but here the molecular axis is fixed as indicated while the Kinetic Energy Release (KER) varies as shown in the insets to the right. High KER corresponds to small inter-nuclear distances $\Delta R$ at the instant of photon absorption as described in figure 1. The dashed lines show a fit with spherical harmonics $(I \in[1,4], m \in[0,1])$. The blue solid line represents the result from a single centre expansion of the molecular ground state and a Convergent Close-Coupling (CCC) expansion of the final two-electron continuum. 